\title{
Computational Model of Edge Effects in Graphene Nanoribbon Transistors
}

\author{
Pei Zhao', Mihir Choudhury ${ }^{2}$, Kartik Mohanram², and Jing Guo ${ }^{1}(\bowtie)$ \\ ${ }^{1}$ Department of Electrical and Computer Engineering, University of Florida, Gainesville, FL 32611, USA \\ ${ }^{2}$ Department of Electrical and Computer Engineering, Rice University, Houston, TX 77005, USA \\ Received: 16 August 2008/ Revised: 13 September 2008/ Accepted: 14 September 2008 \\ CTsinghua Press and Springer-Verlag 2008. This article is published with open access at Springerlink.com
}

\begin{abstract}
We present a semi-analytical model incorporating the effects of edge bond relaxation, the third nearest neighbor interactions, and edge scattering in graphene nanoribbon field-effect transistors (GNRFETs) with armchair-edge GNR (AGNR) channels. Unlike carbon nanotubes (CNTs) which do not have edges, the existence of edges in the AGNRs has a significant effect on the quantum capacitance and ballistic $I-V$ characteristics of GNRFETs. For an AGNR with an index of $m=3 p$, the band gap decreases and the ON current increases whereas for an AGNR with an index of $m=3 p+1$, the quantum capacitance increases and the ON current decreases. The effect of edge scattering, which reduces the ON current, is also included in the model.
\end{abstract}

\section{KEYWORDS}

Graphene nanoribbon field-effect transistor, edge bond relaxation, third nearest neighbor interaction, edge scattering

\section{Introduction}

Graphene [1-3], which is a monolayer of carbon atoms packed into a two-dimensional honeycomb lattice, has been experimentally demonstrated to possess remarkable carrier transport properties. The high mobility and carrier velocity in graphene also promises ballistic devices and high switching speeds. Two-dimensional graphene is a semi-metal material without a band gap. A band gap can be obtained by using a narrow strip of graphene called a graphene nanoribbon (GNR). Unlike carbon nanotubes (CNTs), which are mixtures of metallic and semiconducting materials, a recent experiment [4] demonstrated that all sub-10 nm GNRs are semiconducting due to the edge effects, which make them more attractive for electronic device applications.

In this paper, we present a semi-analytical model incorporates the effects of edge bond relaxation, the third nearest neighbor ( $3 \mathrm{NN}$ ) interactions, and edge scattering in GNR field-effect transistors (GNRFETs) with armchair-edge GNR (AGNR) channels. The edge bond relaxation and the $3 \mathrm{NN}$ interactions (Fig. 1) [5-8], which are not pronounced in CNTs, are found to play an important role on the electronic band structure of AGNRs and the characteristics of GNRFETs. Second nearest neighbor (2NN) interactions, which only shift the dispersion relation in the energy direction but do not change the band structure, can be ignored [5, 7]. Depending on the

Address correspondence to guoj@ufl.edu 


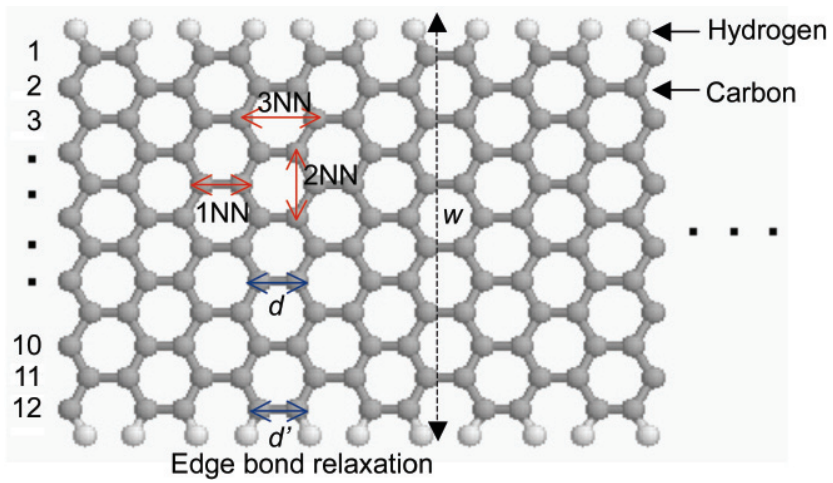

Figure 1 The schematic sketch of an armchair edge GNR (AGNR). The edges of the honeycomb lattice are hydrogen terminated. The edge bonds have a different bond length and bonding parameter from those in the middle of GNR due to edge bond relaxation. The interactions between the first nearest neighbor (1NN), the second nearest neighbor ( $2 \mathrm{NN})$, and the third nearest neighbor (3NN) atoms are also shown

index (width) of the AGNR channel, the edge bond relaxation and the $3 \mathrm{NN}$ interaction effects introduce large differences compared with the simple tight binding (TB) model. For an AGNR with an index of $m=3 p$, the band gap decreases and the ON current increases whereas for an AGNR with an index of $m=3 p+1$, the quantum capacitance increases and the ON current decreases. The effect of edge scattering, which reduces the ON current, is also included in the model described in this paper.

\section{Approach}

The effects of edge bond relaxation, the $3 \mathrm{NN}$ interactions, and edge scattering, all of which are pronounced in GNR field-effect transistors (GNRFETs) but not in CNTFETs, are modeled by extending a top-of-barrier transistor model $[9,10]$, as shown in Fig. 2. At the ballistic limit, the semi-classical model computes the performance limits of the transistor. The Landauer approach can also account for the effect of edge scattering by computing the transmission coefficient of the channel [10]. This model focuses on carrier transport and selfconsistent electrostatics at the top of the potential barrier in the channel, as shown in Fig. 2. It captures the quantum capacitance effect and self-consistent electrostatics in a nanoscale field-effect transistor (FET). The model has been previously used to simulate Si metal oxide semiconductor field-effect transistors (MOSFETs), CNTFETs, and nanowire FETs [9-11]. The performance limits of GNRFETs have been previously assessed using a simple TB model that did not consider edge effects $[12,13]$. The tunneling current is also not considered. However, validation using detailed nonequilibrium Green's function (NEGF) simulations indicates that the semi-classical approach is valid for MOSFET device structures [14] in the simulated bias ranges if the channel length is longer than $20 \mathrm{~nm}$. Recent $\mathrm{ab}$ initio density functional calculations of AGNR band structure indicate the important roles of the edge bond relaxation and the 3NN interactions. Atomistic simulations of GNRFETs using the NEGF formalism have also been reported [15-18]. However, they are computationally expensive and motivate the need for analytical modeling that can encapsulate these effects.

An accurate calculation of the band structure is a necessary input for the model described in this paper. For CNTs, a simple $\mathrm{p}_{\mathrm{z}}$ orbital tight binding (TB) calculation with the first nearest neighbor interaction yields an accurate $E-k$ relation in the energy range relevant for carrier transport. In AGNRs, this calculation, however, fails to yield even a correct band gap, as indicated by comparing the TB results to the $a b$ initio simulation results [5-7]. In an AGNR, the difference is due to both the edge bond

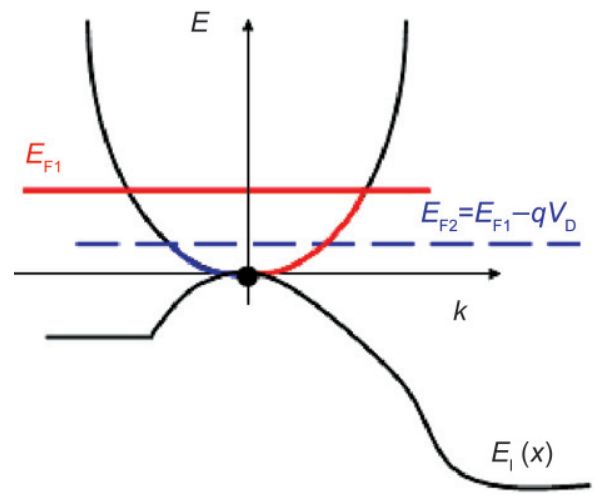

(a)

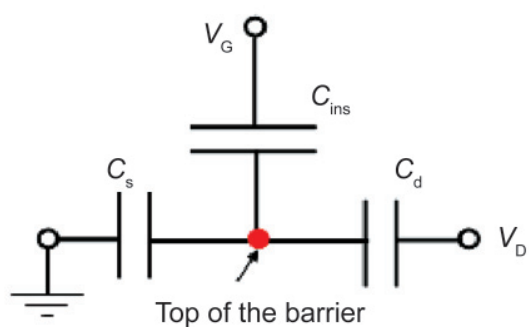

(b)

Figure 2 The transistor model: (a) the first sub-band profile $E_{1}(x)$ and the population at the top of the potential barrier at the ballistic limit; (b) the capacitance model for computation of the self-consistent potential at the top of the barrier 
relaxation and the $3 \mathrm{NN}$ interaction, which a simple TB calculation does not include. A reparameterization of the TB model by introducing additional parameters describing these effects, however, can yield band structures in agreement with the ab initio calculations in the energy range of interest [5]. The $E-k$ dispersion can be expressed as

where

$$
E=\sqrt{(\Delta / 2)^{2}+\left(\hbar v_{s} k\right)^{2}}
$$

$$
\begin{aligned}
\Delta / 2= & \gamma_{1}\left(2 s \cos \frac{p \pi}{m+1}+1\right)+\gamma_{3}\left(2 s \cos \frac{2 p \pi}{m+1}+1\right) \\
& +\frac{4\left(\gamma_{3}+\Delta \gamma_{1}\right)}{m+1} \sin ^{2} \frac{p \pi}{m+1}
\end{aligned}
$$

and

$$
\begin{aligned}
\left(\hbar \gamma_{\mathrm{s}}\right)^{2}= & (3 d)^{2}\left\{-\frac{1}{2} \gamma_{1} s \cos \frac{p \pi}{m+1} \times\left[\gamma_{1}+\gamma_{3}\left(2 \cos \frac{2 p \pi}{m+1}+1\right)\right.\right. \\
& \left.+\frac{4\left(\gamma_{3}+\Delta \gamma_{1}\right)}{m+1} \sin ^{2} \frac{p \pi}{m+1}\right]-\gamma_{3}\left(\gamma_{1}+2 \gamma_{3} \cos \frac{2 p \pi}{m+1}\right. \\
& \left.\left.+\frac{4\left(\gamma_{3}+\Delta \gamma_{1}\right)}{m+1} \sin ^{2} \frac{p \pi}{m+1}\right)\right\}
\end{aligned}
$$

Here, $\gamma_{1}=-3.2 \mathrm{eV}$ and $\gamma_{3}=-0.3 \mathrm{eV}$ are the nearest neighbor and third nearest neighbor hopping parameters, respectively, $\Delta \gamma_{1}=-0.2 \mathrm{eV}$ is the correction of $\gamma_{1}$ for the bonds due to the edge bond relaxation effect [5], and $\hbar$ is the reduced Planck constant.

The band structure-limited velocity is proportional to the slope of $E(k)$ as $v=(1 / \hbar)(\mathrm{d} E / \mathrm{d} k)$. For each onedimensional (1-D) sub-band of a AGNR, the densityof-states (DOS) is inversely proportional to the velocity as $D(E)=4 / h v$. At the ballistic performance limit, the nanotransistor model indicates that the charge density at the top of the potential barrier, $\varepsilon_{\text {top }}$ is

$$
\begin{aligned}
N= & \int_{-\infty}^{+\infty} \frac{1}{2}\left[D\left(E-\varepsilon_{\text {top }}-\Delta / 2\right) f\left(E-E_{\mathrm{F}}\right)+D\left(E-\varepsilon_{\text {top }}-\Delta / 2\right)\right. \\
& \left.\times f\left(E-E_{\mathrm{F}}-q V_{\mathrm{D}}\right)\right] \mathrm{d} E
\end{aligned}
$$

Where the first part comprises positive velocity states filled by the carriers injected from the source, and the second part comprises negative velocity states filled by the carriers from the drain.

Based on a 2-D capacitance model [10], the electrostatics equation is

$$
\varepsilon_{\text {top }}=-q \frac{C_{\text {ins }} V_{\mathrm{G}}+C_{\mathrm{D}} V_{\mathrm{D}}-q N}{C_{\text {ins }}+C_{\mathrm{S}}+C_{\mathrm{D}}}
$$

where $C_{\text {ins }}$ is the gate insulator capacitance and $C_{S}\left(C_{D}\right)$ is the phenomenological source (drain) capacitance, the inclusion of which is necessary to treat the 2-D electrostatic short channel effects.

The current at the ballistic limits is computed as

$I=\frac{2 q k_{\mathrm{B}} T}{h}\left[\ln \left(1+\mathrm{e}^{\frac{E_{\mathrm{F}}-\varepsilon_{\text {top }}}{k_{\mathrm{B}} T}}\right)-\ln \left(1+\mathrm{e}^{\frac{E_{\mathrm{F}}-\varepsilon_{\text {top }}-q V_{\mathrm{D}}}{k_{\mathrm{B}} T}}\right)\right]$

Edge scattering [19] and optical phonon (OP) scattering have been shown to play an important role in GNRFETs. The carrier scattering is treated in a similar way as described in Ref. [10] by computing the transmission coefficient $T$ as

$$
T=\left\{\begin{array}{cl}
\lambda_{\text {edge }} /\left(\lambda_{\text {edge }}+L_{\text {ch }}\right) & \text { if } q V_{\mathrm{D}}<\hbar \omega_{\text {op }} \\
\frac{\lambda_{\text {edge }}}{\lambda_{\text {edge }}+\left(\hbar \omega_{\text {op }} / q V_{\mathrm{D}}\right) L_{\mathrm{ch}}} & \text { if } q V_{\mathrm{D}}>\hbar \omega_{\text {op }}
\end{array}\right.
$$

where $L_{\mathrm{ch}}$ is the channel length of the GNRFET, $\hbar \omega_{\mathrm{op}}$ $\approx 0.18 \mathrm{eV}$ [20] is the OP energy, and $\lambda_{\text {edge }}=15 \mathrm{~nm}$ [19] is the edge scattering mean free path $(\mathrm{mfp}) . \lambda_{\text {edge }}$ is infinitely long for a perfect AGNR edge and of the order of AGNR width for a rough AGNR edge. Under low drain bias, scattering in the channel can cause a carrier to return to the source region and transmission decreases. Under high drain bias, within an approximate critical length of $l=\left(\hbar \omega_{\text {op }} /\right.$ $\left.q V_{\mathrm{D}}\right) L_{\mathrm{ch}}$ near the source end of the channel [21], the back-scattered carriers can return to the source after scattering. Beyond the critical distance, the scattered carriers in the channel near the drain side, however, will be absorbed by the drain without having a direct effect on the source-drain current [19].

\section{Results and discussion}

The electronic properties of the channel material play an important role on transistor characteristics. We first examine the band gap, band structure, density of states, and band structure-limited velocity of an AGNR. In order to examine the effects of edge bond relaxation and the $3 \mathrm{NN}$ interactions, the simulation results are computed using three models. Model 1 is the simple TB model without edge bond relaxation and the $3 \mathrm{NN}$ interactions $\left(\gamma_{3}=\Delta \gamma_{1}=0\right)$. Model 2 considers the edge bond relaxation only $\left(\gamma_{3}=0, \Delta \gamma_{1}\right.$ $=-0.2 \mathrm{eV})$. Model 3 considers both effects $\left(\gamma_{3}=-0.3\right.$ $\mathrm{eV}, \Delta \gamma_{1}=-0.2 \mathrm{eV}$ ) and yields the most accurate band 
structure in agreement with ab initio calculations.

Figure 3 plots the band gap as a function of the AGNR width. Without considering edge bond relaxation and the $3 \mathrm{NN}$ interactions, Model 1 clearly differentiates AGNRs with different widths into two kinds: metallic $(m=3 p+2)$ and semiconducting $(m=3 p$ and $m=3 p+1)$, which contradicts ab initio simulations that indicate all narrow AGNRs are semiconducting [5-7]. This difference motivates the treatment of edge bond relaxation and the $3 \mathrm{NN}$

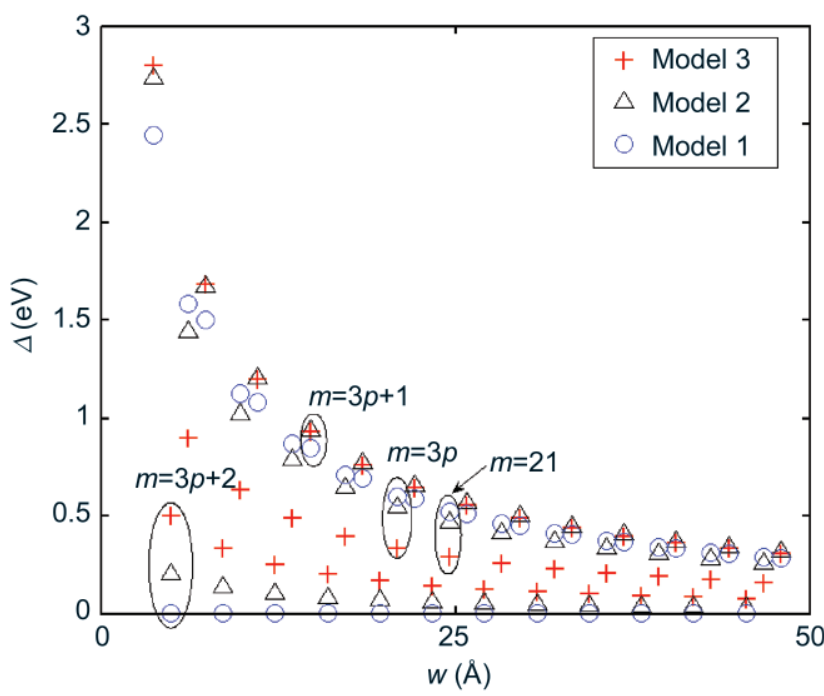

Figure 3 The AGNR band gap as a function of the AGNR width for Model 1, the simple tight binding (TB) model (circles); Model 2, TB with edge bond relaxation only (triangles); and Model 3, TB with both edge bond relaxation and $3 \mathrm{NN}$ interactions (crosses). For the index $m=3 p+1$, the three models predict almost the same behavior. For $m=3 p$, the $3 N N$ interactions plays a more important role than edge bond relaxation. For $m=3 p+2$, both the $3 N N$ interactions and edge bond relaxation have a similar effect on the band gap

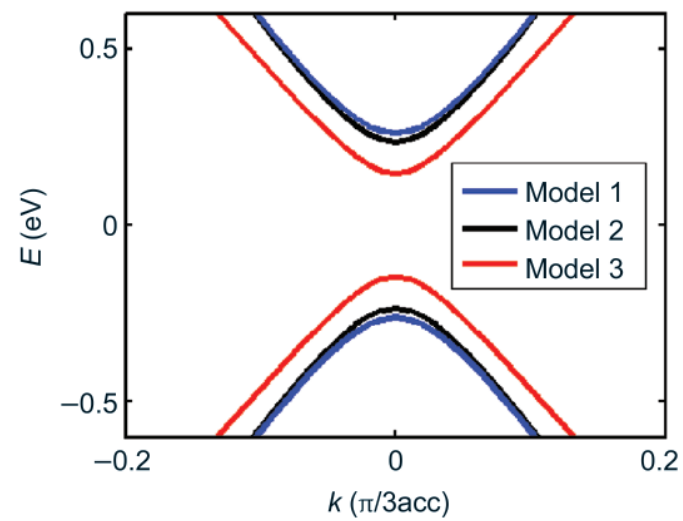

(a) interaction. The simulated energy band gap exhibits three distinct family behaviors. For an AGNR index of $m=3 p+1$, the three models predict similar behavior. For $m=3 p$, the results for simple TB and TB with edge bond relaxation are very close. Thus, the 3NN interactions have the largest effect. For $m=3 p+2$, the simple TB model fails to yield a band gap. Both the $3 \mathrm{NN}$ interaction and the edge bond relaxation are responsible for opening a band gap, with roughly equal contribution from each effect.

Figure $4(\mathrm{a})$ plots the $E-k$ relation for the 21-AGNR $(w=2.46 \mathrm{~nm})$. Comparison between the result obtained from Model 1 (blue lines) and that obtained from Model 2 (black lines) indicates the small effect of the edge bond relaxation on the band structure. The $3 \mathrm{NN}$ interactions, however, have a much more pronounced effect on the band structure. The band gap obtained from Model 1 (blue lines) and that obtained from Model 2 (black lines) are close. A decrease in band gap is clearly observed after the $3 \mathrm{NN}$ interaction is considered using Model 3 (red lines). A plot of the band structure-limited velocity versus energy, which is computed as $v=(1 / \hbar) \mathrm{d} E / \mathrm{d} k$, is shown for the 21-AGNR in Fig. 4(b). From Eq. (1), if $E>>\Delta / 2$ the velocity approaches a constant value. The results indicate that the $3 \mathrm{NN}$ interaction and the edge bond relaxation effects both result in a decrease of the band structure-limited velocity at high energies.

After studying the effects of the $3 \mathrm{NN}$ interaction and edge bond relaxation on the AGNR material

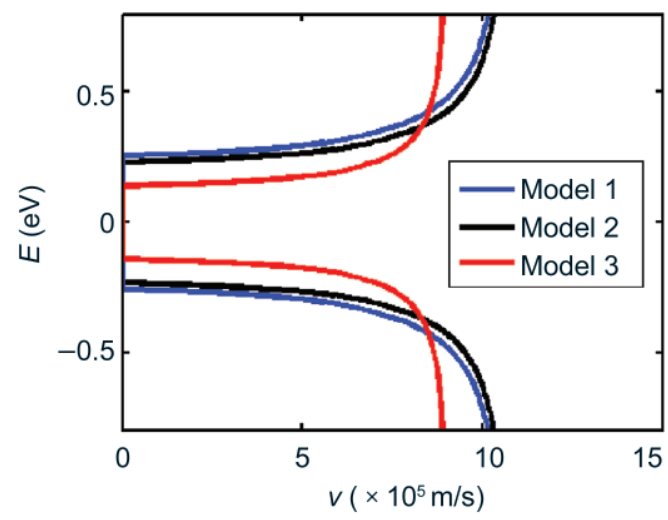

(b)

Figure 4 (a) The first sub-band structure of a 21-AGNR ( $w=2.46 \mathrm{~nm}, \mathrm{acc}=0.142 \mathrm{~nm})$ for the three models, Model 1 (blue lines), Model 2 (black lines), and Model 3 (red lines). Both the 3NN interaction and edge bond relaxation effects result in a decrease in the band gap. However, the effect of the 3NN interactions is larger. (b) The band structure-limited carrier velocity for the first sub-band as a function of energy 
properties, we next examine their effects on device characteristics by using the nanotransistor model. The metal oxide semiconductor (MOS) gate electrostatics of an AGNR are examined first. The gate capacitance, which can be directly characterized by $C-V$ measurements and is important in determining the $I-V$ characteristics, is the series combination of the gate insulator capacitance and the quantum capacitance. We first examine the gate insulator capacitance as a function of the AGNR width and the gate oxide thickness for $\mathrm{SiO}_{2}$ bottomgated GNRFETs as demonstrated experimentally [19, $22,23]$. The gate insulator capacitance $C_{\text {ins }}$ increases linearly as the AGNR width increases because the area of the AGNR increases proportionally, as shown in Fig. 5. Similar to a parallel-plate capacitor, when oxide thickness is reduced, the capacitance increases. The gate capacitance is calculated using Fast Field Solvers [24] with dielectric constant $\varepsilon_{\text {ins }}=3.9$.

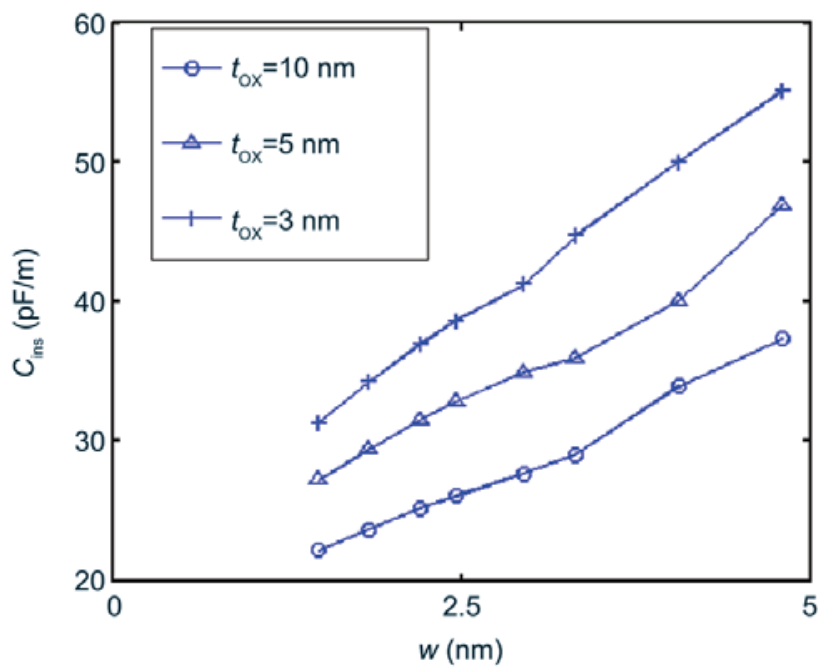

Figure 5 Plot of the gate insulator capacitance versus the AGNR channel width at different oxide thickness of $t_{\mathrm{ox}}=3 \mathrm{~nm}, 5 \mathrm{~nm}$, and 10 nm for a bottom-gated GNRFET

For a given density of states (DOS) $D(E)$, the quantum capacitance $C_{Q}$ of the channel at finite temperature can be calculated [25] as

$$
C_{\mathrm{Q}}=q^{2} \int_{-\infty}^{+\infty} D(E)\left(-\frac{\partial f\left(E-E_{\mathrm{F}}\right)}{\partial E}\right) \mathrm{d} E
$$

Figures 6(a) and (b) show plots of $C_{Q}$ versus the Fermi level $E_{\mathrm{F}}$ with reference to the middle of the AGNR band gap $E_{\mathrm{m}}$ for the three different models at different AGNR widths. The DOS figures shown in Figs. 6(c) and 6(d) are at $T=0 \mathrm{~K}$. A simple expression

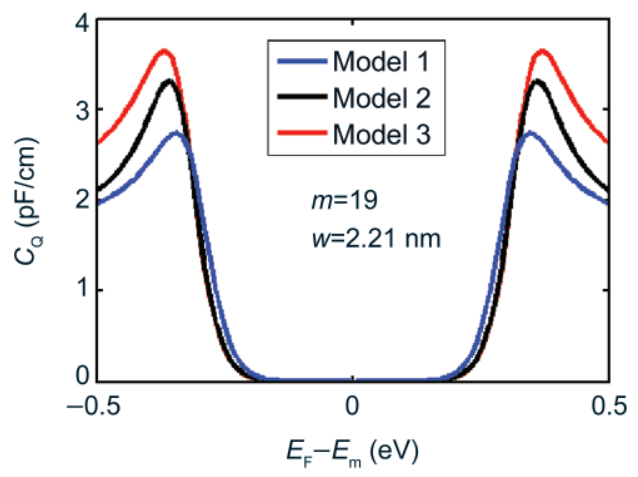

(a)

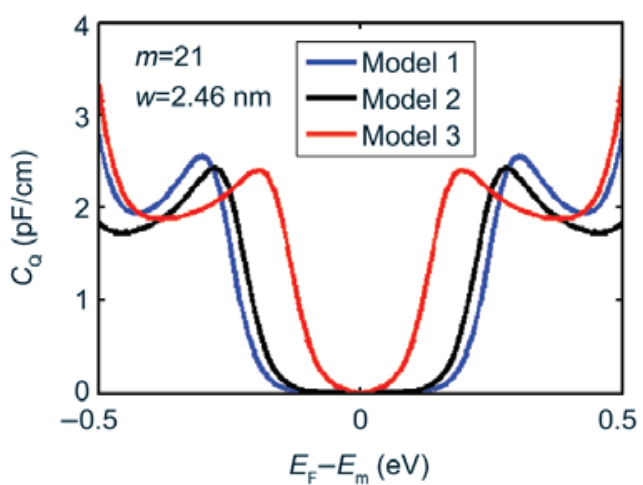

(b)

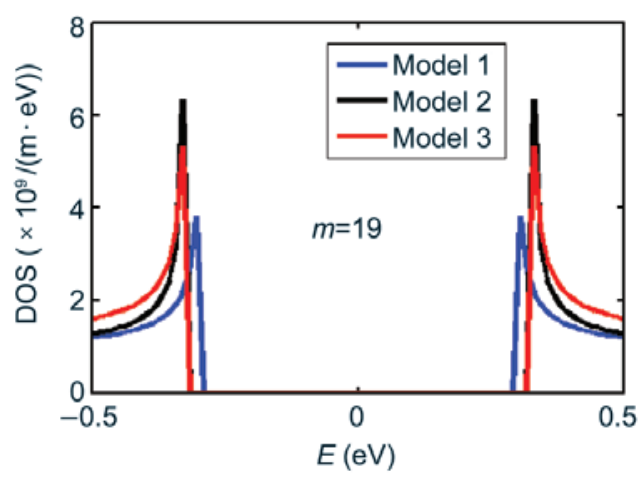

(c)

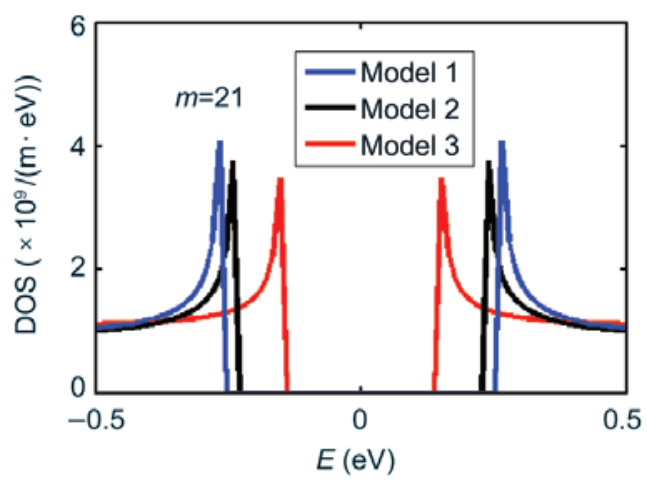

(d)

Figure 6 Plots of the quantum capacitance versus the equilibrium Fermi level for (a) a 19-AGNR and (b) a 21-AGNR. The temperature is $T=300 \mathrm{~K}$. Plots of the DOS versus energy for (c) a 19-AGNR and (d) a 21-AGNR 
for quantum capacitance $C_{\mathrm{Q}}$ at $T=0 \mathrm{~K}$ is $C_{\mathrm{Q}}=q^{2} D(E)$. Equation (8) describes $C_{Q}$ at finite temperature, and the sharp curve with the van Hove singularities of the DOS will be broadened by $k T$ to introduce the effect of temperature.

The band gaps of both the 19-AGNR and the 21-AGNR are larger than $0.5 \mathrm{eV}$, which is much larger than $k T$. Hence, in Figs. 6(a) and (b), the band gap effect can be observed. For the 21-AGNR, the three models give almost the same $C_{Q}$ (Fig. 6(b)) except for Model 3 where the band gap is smaller because it includes both edge bond relaxation and $3 \mathrm{NN}$ interactions. For the 19-AGNR, as shown in Fig. 6(a), the change in band gap is small after the edge effect is included. This leads to a nearly unchanged threshold voltage on the $I-V$ curve. The edge bond relaxation and the $3 \mathrm{NN}$ interactions lead to an increase in the $C_{Q}$ of 19-AGNR, because the edge effects result in a decrease in the band structure-limited velocity and an increase in the DOS in the 19-AGNR as shown in Fig. 6(c).

Figure 7 shows a plot of the charge density as a function of the effective gate voltage $V_{\mathrm{G}}$ for a 21 -AGNR MOS capacitor for the three AGNR band structure models. The results indicate the important role of edge effects on the MOS electrostatics of AGNRs. Including the edge bond relaxation results in a slight decrease in the threshold voltage $V_{\mathrm{T}}$, and including the $3 \mathrm{NN}$ interaction results in a further and larger decrease in $V_{\mathrm{T}}$ because of the decreasing AGNR band gap.

Next we compare the ballistic $I-V$ characteristics

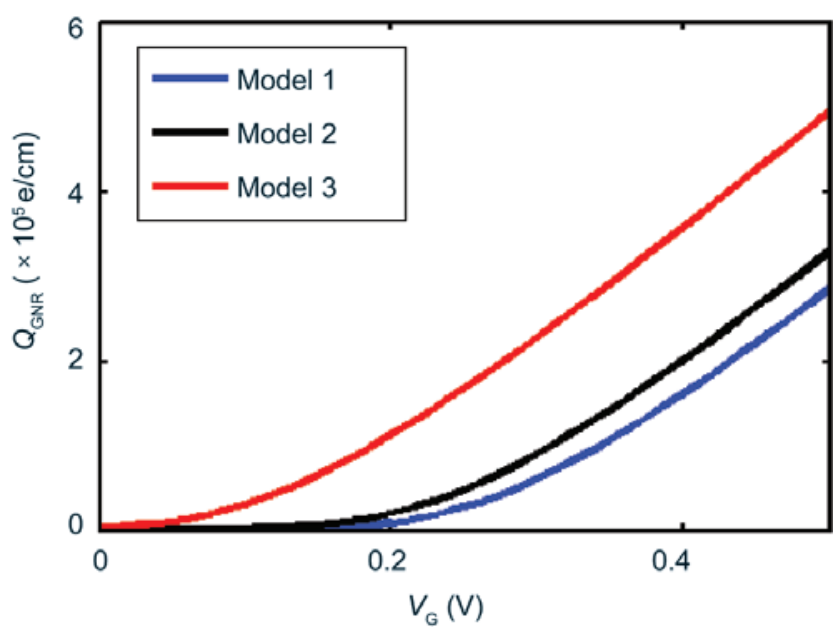

Figure 7 Plots of the charge density as a function of the gate voltage at equilibrium $\left(V_{D}=0\right)$ for a $21-A G N R(w=2.46 \mathrm{~nm})$. The gate insulator capacitance is $26 \mathrm{pF} / \mathrm{m}$, which is for a $10 \mathrm{~nm} \mathrm{SiO}{ }_{2}$ bottom gate as shown in Fig. 5 for two GNRFETs, one with a 19-AGNR channel and the other with a 21-AGNR channel. The 19-AGNR and 21-AGNR are representative of AGNRs with an index of $m=3 p+1$ and $m=3 p$, respectively. Whereas the $3 \mathrm{NN}$ interaction and edge bond relaxation have a large effect on both FETs, opposite trends are observed.

For the 21-AGNR FET, including edge bond relaxation and $3 \mathrm{NN}$ interaction increases the $\mathrm{ON}$ current by $40 \%$. However, for the 19-AGNR FET, including edge bond relaxation and $3 \mathrm{NN}$ interaction results in a decrease in the ON current by $30 \%$. The increase in the ON current in the 21-AGNR FET is mainly due to the decrease in the band gap, which manifests itself as a decrease in $V_{\mathrm{T}}$ as shown in Fig. 8 (b). In contrast, as pointed out in Fig. 3, the edge bond relaxation and the $3 \mathrm{NN}$ interactions have a much smaller effect on the band gap for an AGNR in the $m=3 p+1$ group. The threshold voltage, therefore,

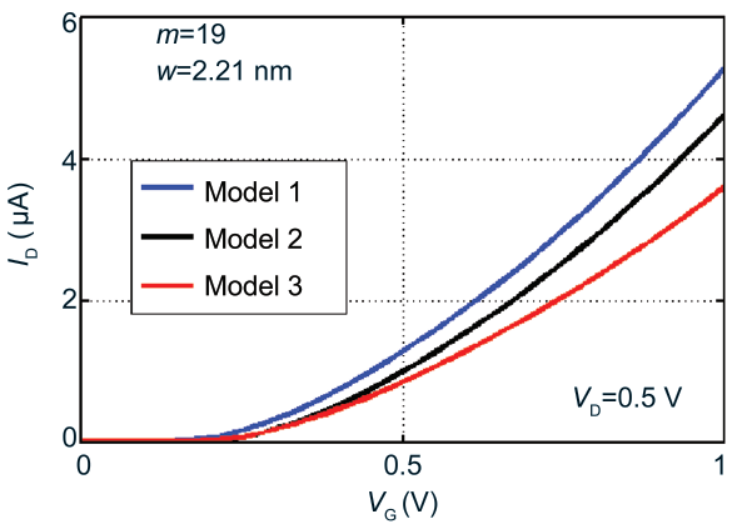

(a)

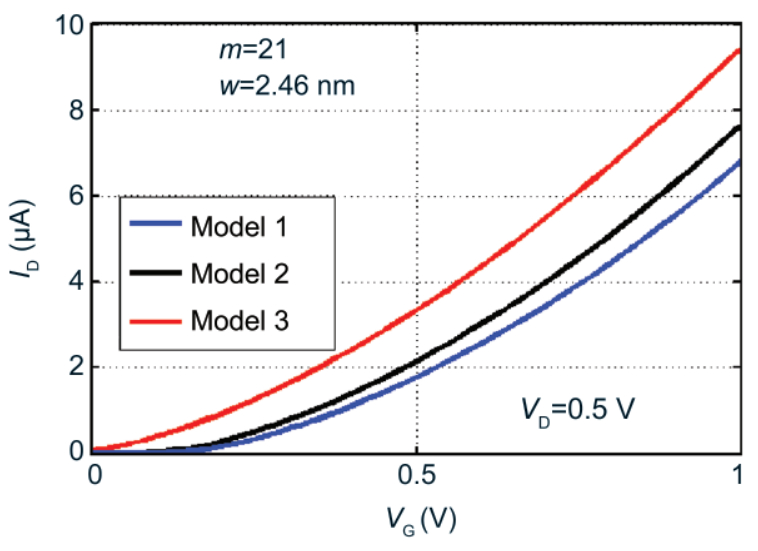

(b)

Figure 8 The ballistic $I_{D}-V_{G}$ characteristics for (a) a 19-AGNR ( $W=2.21$ $\mathrm{nm})$ and (b) a 21-AGNR ( $w=2.46 \mathrm{~nm})$. The gate insulator capacitance is $C_{\text {ins }}=26 \mathrm{pF} / \mathrm{m}$ and $C_{S}=C_{D}=5 \mathrm{pF} / \mathrm{m}$ 
remains approximately the same after the edge effects are considered, as shown in Fig. 8(a).

In spite the small effect on the band gap, the edge effects result in a decrease in the carrier velocity and an increase in the DOS for an AGNR in the $m=3 p+1$ group, which increases the quantum capacitance of the AGNR channel as shown in Fig. 6(a). The gate voltage modulates the top of the barrier less efficiently due to the increase in the AGNR quantum capacitance, resulting in a decrease in the ON current, as shown in Fig. 9(a). In contrast, the decrease in the threshold voltage after the edge effects are included leads to an increase in the ON current for the $m=3 p$ group, as shown in Fig. 9(b).

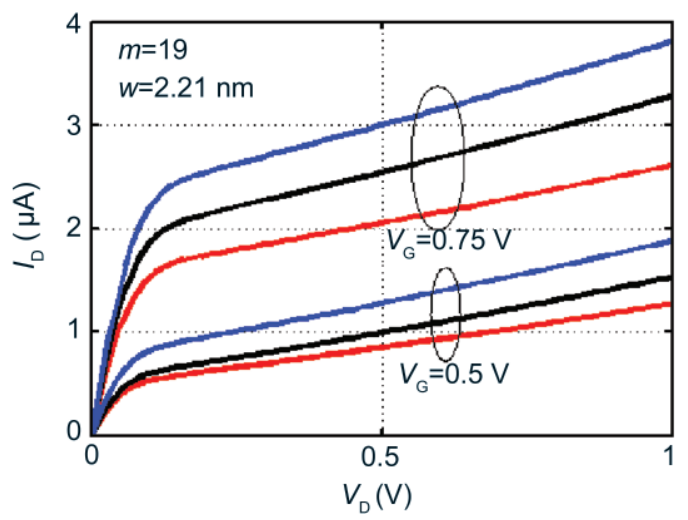

(a)

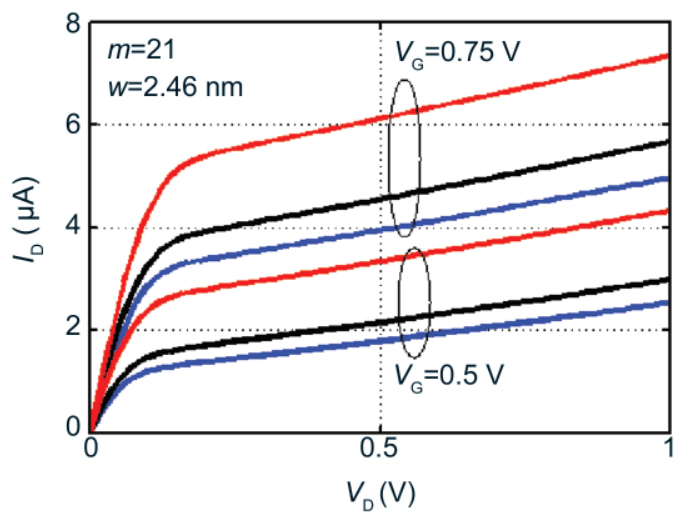

(b)

Figure 9 The ballistic $I_{D}-V_{D}$ characteristics for (a) a 19-AGNR ( $W=2.21$ $\mathrm{nm})$ and (b) a 21-AGNR ( $w=2.46 \mathrm{~nm})$. The gate insulator capacitance is $C_{\text {ins }}=26 \mathrm{pF} / \mathrm{m}$ and $C_{S}=C_{D}=5 \mathrm{pF} / \mathrm{m}$. The legend follows that used in Fig. 4

The $I-V$ characteristics shown in Figs. 8 and 9 are based on an ideal ballistic transport approximation. We next consider the effect of scattering. Figure 10 compares the $I_{\mathrm{D}}-V_{\mathrm{D}}$ characteristics in the presence of edge scattering with a scattering mean free path of
$15 \mathrm{~nm}$ to the ballistic performance limit. The current at $V_{\mathrm{D}}=1 \mathrm{~V}$ is about $31 \%$ of the ballistic current. These results indicate that the $\mathrm{ON}$ current is sensitive to the mean free path of the edge scattering.

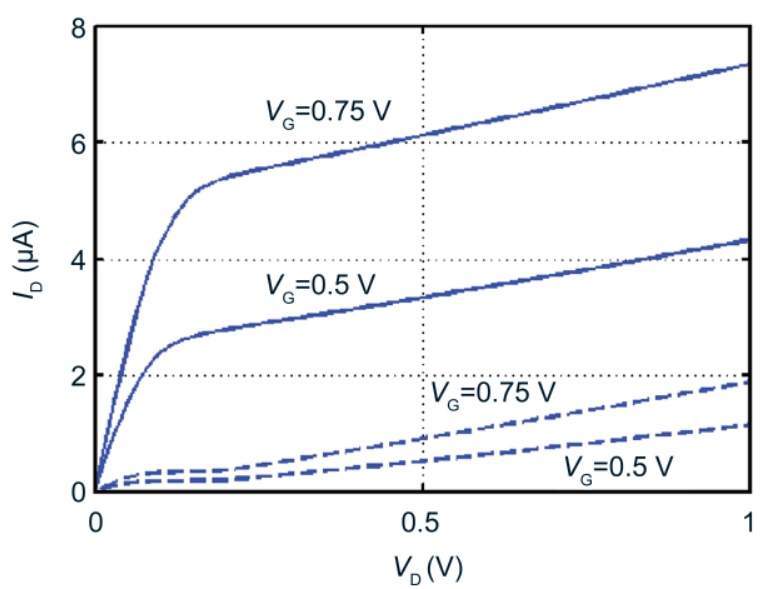

Figure 10 The $I_{D}-V_{D}$ characteristics at $V_{G}=0.5 \mathrm{~V}$ and $0.75 \mathrm{~V}$ in the presence of scattering (dashed lines) is compared with the ideal ballistic approximation (solid lines) for $L_{c h}=100 \mathrm{~nm}$ and $\lambda_{\text {edge }}=15 \mathrm{~nm}$

\section{Conclusions}

This work indicates the important role of edge bond relaxation, which has also been considered in an extended study of GNRFETs [26], the 3NN interactions, and edge scattering in GNRFET characteristics, and shows that these must be considered in future design and optimization. By comparing simulation results with and without including edge effects, we show the important role of the edge effects on the quantum capacitance and $I-V$ characteristics of GNRFETs. For an AGNR with an index of $m=3 p$, the band gap decreases and the ON current increases whereas for an AGNR with an index of $m=3 p+1$, the quantum capacitance increases and the $\mathrm{ON}$ current decreases. The $I-V$ characteristics are also sensitive to the mean free path of the edge scattering.

\section{Acknowledgements}

The authors would like to thank Drs. C. T. White and D. Gunlycke for helpful discussions. This work was supported in part by the Office of Naval Research grant N000140810861, and in part by NSF grants ECCS-0824157 and CCF-0701547. 


\section{References}

[1] Novoselov, K. S.; Geim, A. K.; Morozov, S. V.; Jiang, D.; Zhang, Y.; Dubonos, S. V.; Grigorieva, I. V.; Firsov, A. A. Electric field effect in atomically thin carbon films. Science 2004, 306, 666-669.

[2] Zhang, Y.; Tan, Y. W.; Stormer, H. L.; Kim, P. Experimental observation of the quantum Hall effect and Berry's phase in graphene. Nature 2005, 438, 201-204.

[3] Berger, C.; Song, Z.; Li, X.; Wu, X.; Brown, N.; Naud, C.; Mayou, D.; Li, T.; Hass, J.; Marchenkov, A. N.; Conrad, E. H.; First, P. N.; de Heer, W. A. Electronic confinement and coherence in patterned epitaxial graphene. Science 2006, 312, 1191-1196.

[4] Li, X.; Wang, X.; Zhang, L.; Lee, S.; Dai, H. Chemically derived, ultrasmooth graphene nanoribbon semiconductors. Science 2008, 319, 1229-1232.

[5] Gunlycke, D.; White, C. T. Tight-binding energy dispersions of armchair-edge graphene nanostrips. Phys. Rev. B 2008, 77, 115116.

[6] Son, Y. W.; Cohen, M.; Louie, S. Energy gaps in graphene nanoribbons. Phys. Rev. Lett. 2006, 97, 216803.

[7] White, C. T.; Li, J.; Gunlycke, D.; Mintmire, J. W. Hidden one-electron interactions in carbon nanotubes revealed in graphene nanostrips. Nano Lett. 2007, 7, 825-830.

[8] Sasaki, K.; Murakami, S.; Saito, R. Stabilization mechanism of edge states in graphene. Appl. Phys. Lett. 2006, 88, 113110.

[9] Natori, K. Ballistic metal-oxide-semiconductor fieldeffect transistor. J. Appl. Phys. 1994, 76, 4879-4890.

[10] Lundstrom, M.; Guo, J. Nanoscale Transistors: Device Physics, Modeling and Simulation; Springer: New York, 2006.

[11] Wong, H. -S. P.; Deng, J.; Hazeghi, A. Krishnamohan, T.; Wan, G. C. Carbon nanotube transistor circuits: Models and tools for design and performance optimization. Proc. Intl. Conf. Computer-aided Design 2006, p. 651.

[12] Ouyang, Y.; Yoon, Y.; Fodor, J.; Guo, J. Comparison of performance limits for carbon nanoribbon and carbon nanotube transistors. Appl. Phys. Lett. 2006, 89, 203107.

[13] Liang, G.; Neophytou, N.; Nikonov, D. E.; Lundstrom, M. Performance projections for ballistic graphene nanoribbon field-effect transistors. IEEE T. Electron Dev. 2007, 54, 677-682.
[14] Rahman, A.; Guo, J.; Datta, S.; Lundstrom, M. Theory of ballistic transistors. IEEE T. Electron Dev. 2003, 50, 1853-1864.

[15] Fiori, G.; Iannaccone, G. Simulation of graphene nanoribbon field effect transistors. IEEE Electr. Device $L$. 2007, 28, 760-762.

[16] Ouyang, Y.; Yoon, Y.; Guo, J. Scaling behaviors of graphene nanoribbon FETs: A three-dimensional quantum simulation study. IEEE T. Electron Dev. 2007, 54, 2223-2231.

[17] Liang, G. C.; Neophytou, N.; Lundstrom, M.; Nikonov, D. E. Ballistic graphene nanoribbon metal-oxidesemiconductor field-effect transistors: A full real-space quantum transport simulation. J. Appl. Phys. 2007, 102, 054307.

[18] Guan, X.; Zhang, M.; Liu, Q.; Yu, Z. Simulation investigation of double-gate CNR-MOSFETs with a fully self-consistent NEGF and TB method. IEDM Tech. Dig. 2007, 761-764.

[19] Wang, X.; Ouyang, Y.; Li, X.; Wang, H.; Guo, J.; Dai, $\mathrm{H}$. Room temperature all semiconducting sub-10 nm graphene nanoribbon FETs. Phys. Rev. Lett. 2008, 100, 206803.

[20] Perebeinos, V.; Tersoff, J.; Avouris, P. Electron-phonon interaction and transport in semiconducting carbon nanotubes. Phys. Rev. Lett. 2005, 94, 086802.

[21] Lundstrom, M. Elementary scattering theory of the Si MOSFET. IEEE Electr. Device L. 1997, 18, 361-363.

[22] Han, M. Y.; Ozyilmaz, B.; Zhang, Y.; Kim, P. Energy bandgap engineering of graphene nanoribbons. Phys. Rev. Lett. 2007, 98, 206805.

[23] Chen, Z. H.; Lin, Y. M.; Rooks, M. J.; Avouris, P. Graphene nanoribbon electronics. Physica E 2007, 40, 228-232.

[24] Fast Field Solvers. http://www.fastfieldsolvers.com (accessed 2008).

[25] Datta, S. Quantum Transport: Atom to Transistor; Cambridge University Press: Cambridge, 2005, pp. 170176.

[26] Yoon, Y.; Fiori, G.; Hong, S.; lannaccone, G.; Guo, J. Performance comparison of graphene nanoribbon FETs with Schottky contacts and doped reservoirs. IEEE T. Electron Dev. 2008, 55, 2314-2323. 